\title{
FOREST RESOURCE MEASUREMENTS BY COMBINATION OF TERRESTRIAL LASER SCANNING AND DRONE USE
}

\author{
K. Cheung a, *, M. Katoh ${ }^{a}$, M. Horisawa ${ }^{b}$ \\ ${ }^{a}$ Institute of Mountain Science, Shinshu University, 8304, Minamiminowa-Vill. Kamiina-County, Nagano, 399-4598, Japan, \\ (17as409g, mkatoh)@shinshu-u.ac.jp \\ b Kita-Shinshu forest owners' cooperative, 938-1 Kabeta, Nakano, Nagano, 399-4598, Japan, m.horisawa@jforest- \\ kitashinshu.or.jp
}

\section{Commission III, WG III/1}

KEY WORDS: Terrestrial Laser Scanning, Drone, Airborne Laser Scanning, Stand Level, Individual Level, 100\% Tree Detection Rate

\begin{abstract}
:
Using terrestrial laser scanning (TLS), forest attributes such as diameter at breast height (DBH) and tree location can be me asured accurately. However, due to low penetration of laser pulses to tree tops, tree height measurements are typically underestimated. In this study, data acquired by TLS and drones were combined; DBH and tree locations were determined by TLS, and tree heights were measured by drone use. The average tree height error and root mean square error (RMSE) of tree height were 0.8 and $1.2 \mathrm{~m}$, respectively, for the combined method, and -0.4 and $1.7 \mathrm{~m}$ using TLS alone. The tree height difference was compared using airborne laser scanning (ALS). Furthermore, a method to acquire $100 \%$ tree detection rate based on TLS data is suggested in this study.
\end{abstract}

\section{INTRODUCTION}

Terrestrial laser scanning (TLS) has great potential to be applied in operational forestry management. Millimeter-level detail can be acquired by TLS (Liang, 2016). TLS has been used to retrieve forest inventory variables for an urban heterogeneous forest (Moskal, 2012) and to estimate the timber quality of Scots pine (Kankare, 2014). TLS has also been used to map tree locations to train airborne laser scanning (ALS) data (Lindberg, 2012; Vastaranta, 2014). However, although TLS provides more accurate data than do manual field measurements and other laser scanning methods such as ALS, TLS can seldom provide a $100 \%$ tree detection rate due to its own deficiencies. TLS-derived tree heights can underestimate field-measured heights when shadowing is prevalent (Srinivasan, 2015). In this study, we compared TLS measurements to ALS measurements with respect to tree detection rate and diameter at breast height (DBH) estimation using a manual field inventory as reference data. Respect to tree height measurement, a combination method of TLS and drone use developed in this study was compared to TLS measurement using ALS data as reference data. Furthermore, a method to acquire $100 \%$ tree detection rate based on TLS data is suggested in this study.

\section{METHODS AND MATERIALS}

\subsection{Study area}

The study area is a planted Japanese cedar (Cryptomeria japonica) forest located in Kakuma, Yamanouchi-chou, Nagano-ken, Japan $\left(36^{\circ} \mathrm{N}, 138^{\circ} \mathrm{E}\right)$. The forest was 59 years old at the time of TLS measurement. An $80 \times 80-\mathrm{m}$ plot was created for this study, including a total of 511 Japanese cedars; other species were not considered because they are not harvested. The average slope of the plot was $11.8^{\circ}$.

\subsection{TLS measurement and data analysis}

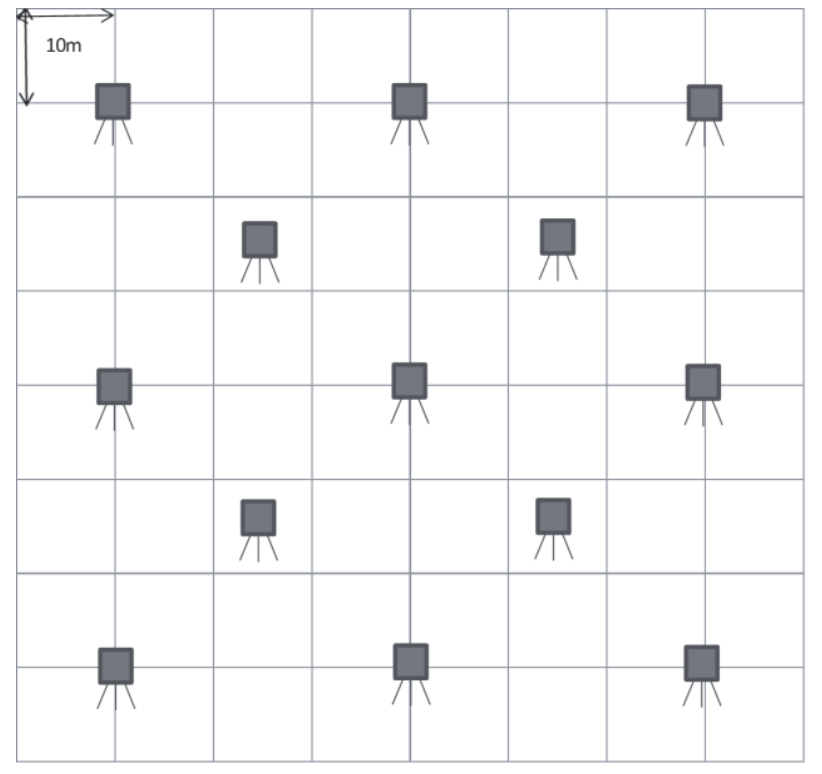

TLS scanner

Figure 1. Locations of terrestrial laser scanning (TLS) measurements within the plot

\footnotetext{
* Corresponding author
} 
We performed 13 TLS measurements in the plot in August 2016. The locations of these measurements are shown in Figure 1. The TLS scanner used in this study was the Laser Scanner Focus3D X 130 (FARO, USA). TLS measurements were recorded using the SCENE 5.2 software (FARO, USA). Individual tree analysis of TLS data was performed using the Digital Forest (Woodinfo, Japan) software. The results were mapped using the ArcGIS 10.0 software (ESRI, Japan). A digital elevation model (DEM) was extracted from the TLS data using TerraScan and TerraModeler software (TerraSolid, Finland).

\subsection{Drone measurement}

Drone measurements were conducted in August 2016. The drone used in this study was the Phantom 3 Professional (DJI, China). The route of the drone flight was calculated such that both the overlap rate and side-lap rate exceeded $80 \%$ for adjacent photographs. The flight altitude was set to $75 \mathrm{~m}$ above the ground. In total, 96 photographs were used in this study. Ortho images and a digital surface model (DSM) were prepared using the PhotoScan Professional software (Agisoft, Russia).

\subsection{Manual field inventory}

Manual field measurements were collected for the entire plot in August, 2016, based on the TLS measurement results. Trees not detected by TLS were added, and over-detected trees from the TLS data were deleted so that the dataset was an exact representation of the number of trees in the plot. Every tree was numbered by attaching number tapes to the trunks. The DBH of every counted tree was measured using a measuring tape.

\subsection{ALS data}

ALS measurement was conducted in June, 2013. ALS data were provided to, and analyzed by, Asia Air Survey Co., Ltd. The DBH results from the TLS data were compared with those from the ALS data, using manual field data as a reference. Individual tree heights from the TLS data were compared with those obtained from the combined method of TLS and drone use, using ALS data as a reference.

\subsection{Combination of TLS and drone use}

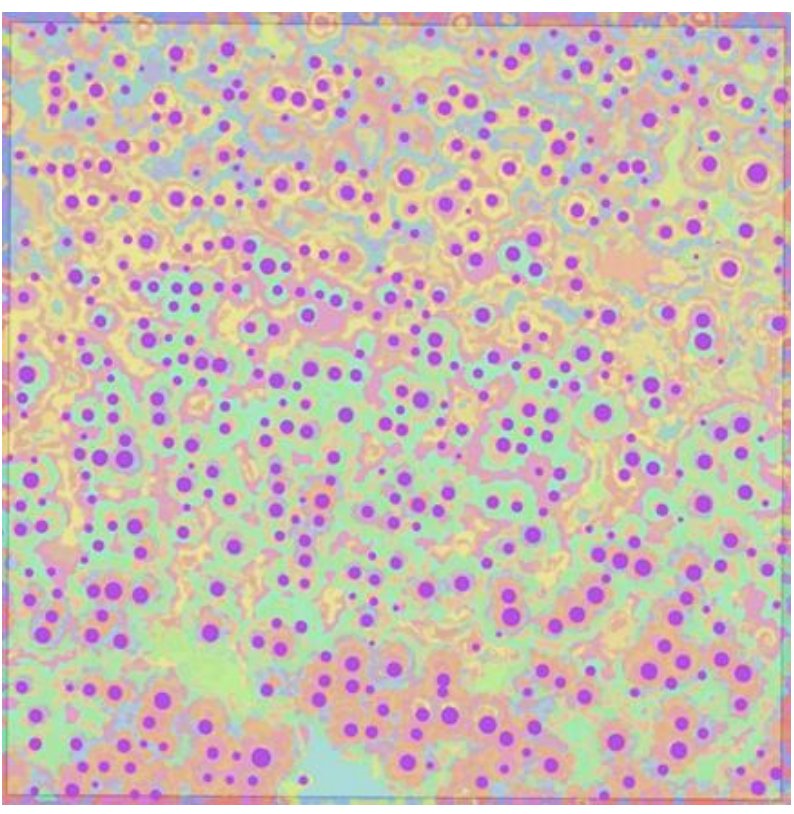

: TLS individual tree

Figure 2. Map of trees within the plot created using a combination of data collected by TLS and drone use

The TLS scanner has its own Global Positioning System (GPS) sensor; however, its error is large in dense forest. The accuracy of the drone GPS measurement is typically high due to good satellite signal reception. We performed geometric correction of TLS so that the tree locations determined by TLS fit those determined by the drone. Manual geometric correction was made first and the distance and the angle geometric correction were estimated. The estimations of the distance and the angle geometric correction were used for extra data input such as TLS scanner locations. To visualize the tree heights obtained by the drone, we applied random colour classification to the drone DSM data according to height (Figure 2). Because the tree locations determined by TLS were based on the trunk locations and the tree locations of the drone data were based on the tree tops, some tree locations did not match exactly between the two datasets. The distance and the angle geometric corrections for the TLS data were $10.3 \mathrm{~m}$ and $12.8^{\circ}$, respectively.

\subsection{Individual tree matchings between TLS and ALS data}

Not all individual trees in the plot were extracted by TLS or ALS measurements. To estimate the difference in accuracy between TLS and ALS measurements, individual tree matchings were needed. In this study, 1) tree distance, 2) pattern of tree locations, 3) DBH were considered in the individual tree matchings. Trees that were not detected by both methods were excluded from the individual-level comparisons between TLS and ALS data.

\subsection{Tree height compensation in the combination method}

To combine TLS and drone use, we calculated tree heights by subtracting the TLS DEM data from the drone DSM data. Due to a gap in altitude values between the two measurements, tree 
height compensations were required. We chose 10 sample trees whose tree tops were clearly identified in the plot, and determined their tree heights using Vertex IV (Haglof, Sweden). The average difference between the sample trees and the corresponding TLS-measured trees was then calculated, and this difference was applied to all of the extracted trees in the plot.

\section{RESULT}

\subsection{Tree detection}

\begin{tabular}{|l|r|r|}
\hline & TLS & ALS \\
\hline Number of trees detected & 514 & 387 \\
\hline Detection rate \% & 100.6 & 75.7 \\
\hline Corresponding trees & 493 & 356 \\
\hline Corresponding detection rate \% & 96.5 & 69.7 \\
\hline Over-detected trees & 21 & 23 \\
Non-detected trees & 18 & 155 \\
Uncounted trees & 0 & 8 \\
\hline Field Data = 511 & & \\
\hline
\end{tabular}

Table 1. Tree detection rates obtained by terrestrial laser scanning (TLS) and airborne laser scanning (ALS)

The tree detection rates of TLS and ALS are listed in Table 1. In total, there were 511 trees in the plot; 514 trees were detected by TLS, and 387 trees by ALS. Over-detection existed for both TLS and ALS. The over- detection was likely due to the detection of other tree species and limitations of the analysis software. To mitigate the influence of over-detection, corresponding detection rate is introduced in this study. With 21 and 23 trees over-detected by TLS and ALS respectively, the corresponding detection rates were 96.5 and $69.7 \%$ for TLS and ALS, respectively. Non-detected trees numbered 18 and 155 for TLS and ALS, respectively, which shows that TLS has extremely high detection power, whereas ALS has limited detection power. Eight uncounted trees were detected by ALS; these were outside of the plot area.

\subsection{DBH}

3.2.1 Stand level: Table 2 shows the comparison of standlevel DBH values from field data, TLS, and ALS. Only trees detected by both TLS and ALS $(n=349)$ were considered. The average DBH values calculated from field data, TLS, and ALS were $32.6,32.5$, and $32.4 \mathrm{~cm}$, respectively, showing that both TLS and ALS provided accurate DBH estimates at the stand level.

\begin{tabular}{|l|l|r|r|r|}
\hline & & Field Data & TLS & ALS \\
\hline Number of trees & & 349 & 349 & 349 \\
\hline DBH $(\mathrm{cm})$ & average & 32.6 & 32.5 & 32.4 \\
& minimum & 13.0 & 13.7 & 12.0 \\
& maximum & 52.5 & 54.8 & 56.0 \\
& sd & 7.0 & 6.9 & 7.7 \\
\hline
\end{tabular}

sd: standard deviation

Table 2. Comparison of stand-level diameter at breast height (DBH) measurements collected in the field, and by TLS and ALS

\begin{tabular}{|l|l|r|r|}
\hline & & TLS & ALS \\
\hline Number of trees & & 349 & 349 \\
\hline DBH error $(\mathrm{cm})$ & bias & -0.1 & -0.2 \\
& minimun & -9.8 & -13.8 \\
& maximum & 9.6 & 11.9 \\
& RMSE & 2.0 & 4.6 \\
\hline
\end{tabular}

RMSE: root mean squared error

Table 3. Comparison of individual-level DBH measurements obtained by TLS and ALS

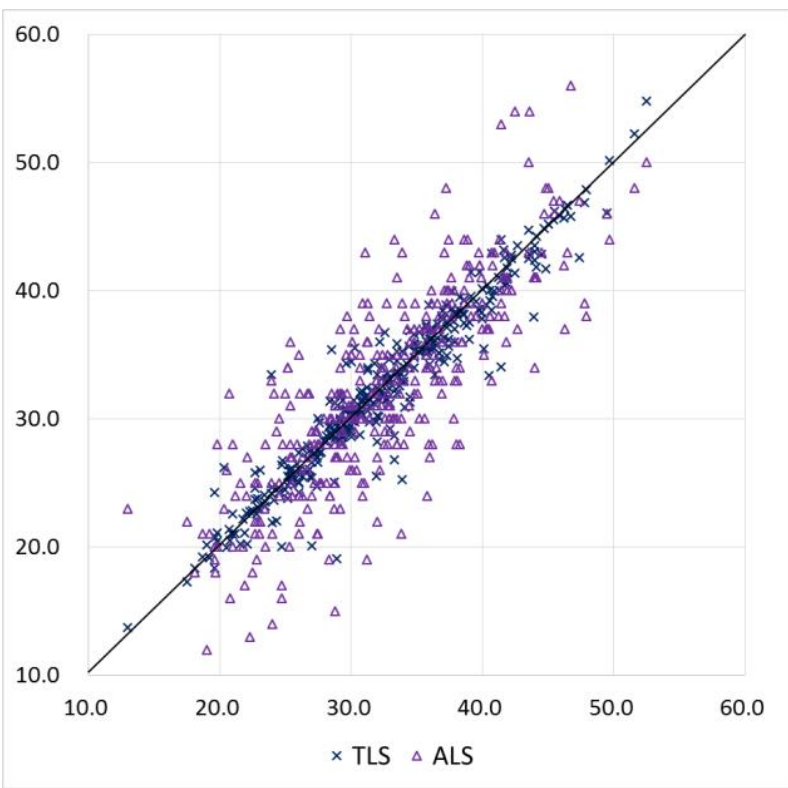

Figure 3. DBH comparison of TLS and ALS for individual tree level

3.2.2 Individual levcl: Table 3 and Figure 3 show the comparison of individual-level DBH measurements obtained by TLS and ALS, using field data as a reference. The RMSE of TLS and ALS were 2.0 and 4.6, respectively, which shows that DBH measurements obtained by TLS were more accurate than those obtained by ALS at the individual level.

\subsection{Tree height}

3.3.1 Stand level: Table 4 shows the comparison of standlevel tree height measurements obtained by ALS, TLS, and the combination of TLS and drones. The average tree height estimated by ALS, TLS, and the combination method were 25.1, 24.7 and $25.9 \mathrm{~m}$, respectively. Using ALS data as a reference, we determined that tree heights obtained by TLS were underestimated, whereas those obtained by the combination method were overestimated. In the combination method, the heights of 10 sample trees were measured to determine tree height compensation. Table 5 shows the comparison of the average tree height of the sample trees and the corresponding heights determined by ALS. One tree was not represented in the ALS dataset due to non-detection. The average tree height of the sample trees was $26.0 \mathrm{~m}$, which was $0.9 \mathrm{~m}$ higher than the value obtained by ALS. If this difference is considered, the tree height estimate determined by the combination method approaches that obtained by ALS. 


\begin{tabular}{|l|l|r|r|r|}
\hline & & ALS & TLS & Combination \\
\hline Number of trees & & 349 & 349 & 349 \\
\hline Tree height $(\mathrm{m})$ & average & 25.1 & 24.7 & 25.9 \\
& minimum & 19.0 & 11.4 & 18.2 \\
& maximum & 32.7 & 31.9 & 32.8 \\
\hline & sd & 2.6 & 2.4 & 2.5 \\
\hline
\end{tabular}

Table 4. Comparison of tree height measurements obtained by ALS, TLS, and the combination method

\begin{tabular}{|l|r|r|}
\hline & ALS & Sample trees \\
\hline Number of trees & 9 & 9 \\
\hline average tree height $(\mathrm{m})$ & 25.1 & 26.0 \\
\hline
\end{tabular}

Table 5. Comparison of average tree height, measured by ALS, with sample trees

3.3.2 Individual level: Table 6 and Figure 4 show a comparison of individual-level tree height measurements obtained by TLS and by the combination method, using ALS data as a reference. The tree height RMSEs for data obtained by TLS and the combination method were 1.7 and 1.2, respectively, showing that tree height estimation by the combination method was better than that by TLS alone.

\begin{tabular}{|l|l|r|r|}
\hline & & \multicolumn{1}{|l|}{ TLS } & Combination \\
\hline Tree height error $(\mathrm{m})$ & average & -0.4 & 0.8 \\
& minimum & -14.6 & -3.0 \\
& maximum & 7.6 & 3.6 \\
& RMSE & 1.7 & 1.2 \\
\hline
\end{tabular}

Table 6. Comparison of individual-level tree height measurements obtained by TLS and the combination method

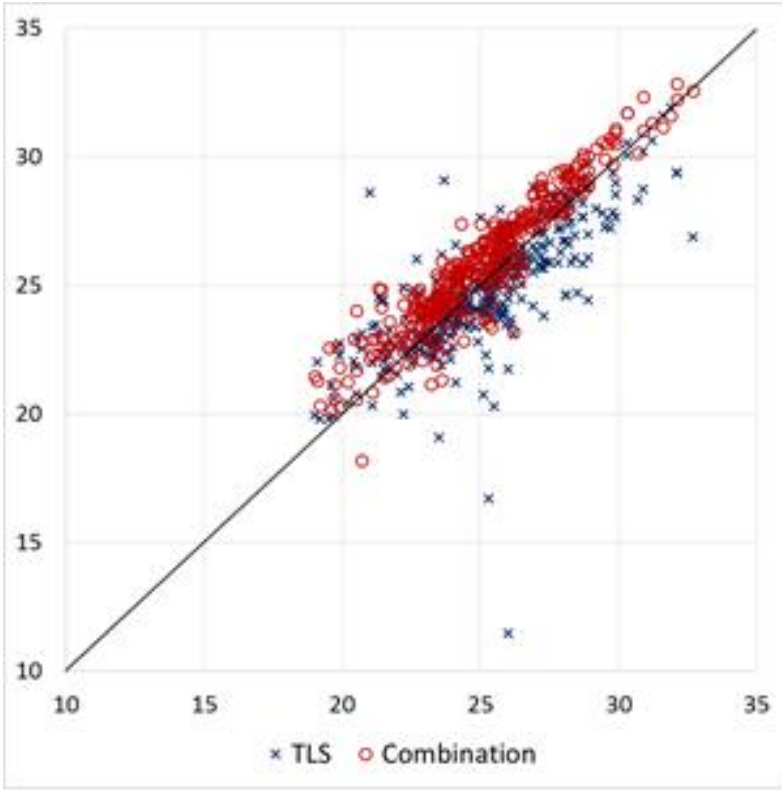

Figure 4. Comparison of individual-level tree height error estimated from data obtained by TLS and the combination method

\section{DISCUSSION}

\subsection{Further field verification of TLS measurement}

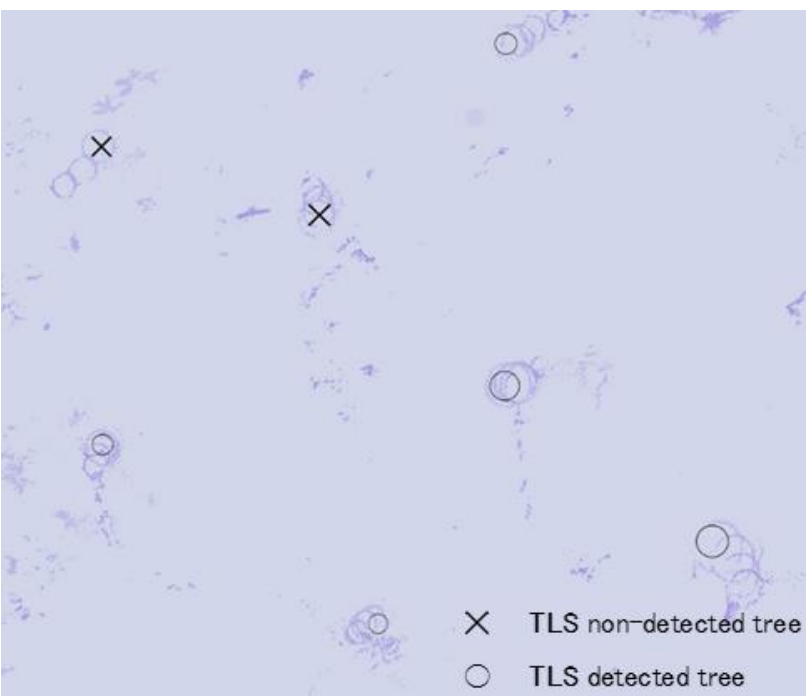

Figure 5. Sliced point clouds representing detected and nondetected trees

TLS measurement had a corresponding detection rate exceeding 95\%. However, the TLS dataset contained some over-detected and non-detected trees. Due to its high detection rate and location accuracy, it would be desirable to perform field identification of over-detected and non-detected trees. Some trees were not detected due to parameter settings of the analysis software. The locations of non-detected trees can be identified from sliced point clouds (Figure 5). To acquire reference data with all the required trees included, TLS is a very powerful but not perfect method. To ensure that all required trees are measured, we recommend field verification; this measure is not recommended for ALS data, due to the extreme difficulty of identifying the large numbers of overdetected and non-detected trees and location errors introduced by the discrepancy between tree top and tree trunk locations for the same trees.

\subsection{Validity of the combination of TLS and drone use}

Tree height estimates derived from a combination of TLS and drone use were overestimated compared to the ALS-derived tree heights, for two reasons. First, ALS measurements were conducted in 2013, whereas TLS and drone measurements were conducted in 2016. Trees may have grown during this period. Second, our tree compensation method may have been inaccurate. We used Vertex IV to measure the heights of 10 sample trees whose tree tops were clearly identified. However, there may have been errors in the manual measurements. If the offset is properly corrected, the tree height estimation made by the combination of TLS and drone use can eliminate underestimation in TLS measurements caused by the difficulty of laser penetration of the tree crowns to the tree tops.

\section{CONCLUSION}

TLS measurements provided highly accurate DBH and location estimates. With further field verification, a $100 \%$ detection 
rate can be achieved. Drone use can also help to improve the accuracy of tree height estimation. In conclusion, measurement by a combination of TLS and drone use can replace manual field measurements, which are costly in terms of financial investment, time, and effort.

\section{REFERENCES}

Kankare, V., et al., 2014. Estimation of the Timber Quality of Scots Pine with Terrestrial Laser Scanning. Forests 2014, 5, 1879-1895.

Liang, X., et al., 2016. Terrestrial laser scanning in forest inventories. ISPRS Journal of Photogrammetry and Remote Sensing 115 (2016) 63-77.

Lindberg, E., et al. 2012. Estimation of stem attributes using a combination of terrestrial and airborne laser scanning. Eur $J$ Forest Res (2012) 131:1917-1931.

Moskal, L.M., et al. 2012. Retrieving Forest Inventory Variables with Terrestrial Laser Scanning (TLS) in Urban Heterogeneous Forest. Remote Sens. 2012, 4, 1-20.

Srinivasan, S., et al. 2015. Terrestrial Laser Scanning as an Effective Tool to Retrieve Tree Level Height, Crown Width, and Stem Diameter. Remote Sens. 2015, 7, 1877-1896.

Vastaranta, M., et al. 2014. Multisource Single-Tree Inventory in the Prediction of Tree Quality Variables and Logging Recoveries. Remote Sens. 2014, 6, 3475-3491. 